$\xi=1$ -

\title{
High Speed Realistic Mobility Model for TN-Multi Lane Highway Environment
}

\author{
Vivitha Varshini R.J ${ }^{1 *}$, Naskath $\mathbf{J}^{2}$, Paramasivan $\mathbf{B}^{3}$ \\ ${ }^{1,2,3}$ National Engineering College, Kovilpatti. \\ *Corresponding author E-mail: vivi.rjv@gmail.com
}

\begin{abstract}
Vehicular Ad-hoc Networks (VANETs) are self-forming networks built from moving vehicles. The most important quality of VANETs is the high mobility of nodes, which makes the mobility model as one of the most important parameters. The mobility pattern in the mobility models is used to simulate the movement of the vehicles, which plays an important role in developing an effective communication for the vehicular network. The challenge is to generate suitable microscopic mobility model from the correct and realistic dataset. In this paper, the work analyses the different type of data sources in terms of various mobility models. Finally, it proposes the effective mobility model for the high speed multi-lane highway scenario. Then the performance metrics are analyzed for this proposed model using mobility and network simulators.
\end{abstract}

Keywords: Lane changing model; Mobility Model; MOVE; Network topology; SUMO; VANET;

\section{Introduction}

The possibility of direct exchange of dynamic data between vehicles over an ad hoc environment called as Vehicular Ad hoc Network (VANET). VANET is a subclass of Mobile Ad Hoc networks (MANETs) to provide the communication among the vehicles which acts as the mobile nodes and the nearby roadside equipment. The vehicles in the vehicular network have been integrated with the new generation wireless networks and provide the effective communication with other nodes using different communicating technologies like Wi-Fi, IEEE b/g, Wi-Max IEEE, Bluetooth, IRA. Dedicated Short Range Communications (DSRC) and IEEE 802.11p Wireless Access for Vehicular Environment (WAVE) have been approved as standards for PHY and MAC layers for the vehicular networks.

Mobility models in the vehicular networks describe the movement pattern of the vehicles that can communicate wirelessly. It depicts the entire facet related to the individual behavior of the vehicle such as speed, acceleration, and behavior of each driver while interacting with other drivers or with infrastructure based on microscopic and macroscopic factors.

For the developing the realistic scenario, the apt mobility model and the optimal time for simulation is needed. Also, an effective characterization of vehicular mobility requires the comparison of both real time data and the simulation data in terms of the various mobility models, resulting in the liveliness of the VANET topology.

This work proposed two traffic and one network simulator for simulation of various mobility models. The mobility simulators[14] MOVE (MObility model generator for VEhicular networks) and SUMO provide trace files for network simulator NS2.From the result of NS2 network parameters are analyzed in terms of various mobility models for the proposed environment.
The design of this paper is to examine the VANET topology features on a multilane highway scenario by fusing the realistic traces of micro mobility obtained using the real world topology and the real world database traffic with the realistic mobility models, taken into the account. For this purpose, the Tuticorin multilane highway environment is taken as a proposed road topology for this mobility based work. The different views of the proposed topology are shown in Figure 1. This paper compares the performance of this realistic situation to the various mobility models using the sundry metrics of interest. The remaining paper is arranged as follows. Section II shows the overview of mobility model and their usages for the vehicular networks. Section III describes proposed work methodology. Section IV describes the performance analysis of the proposed work. Finally, concluding factors and the future work is given in section $\mathrm{V}$.

\section{Related work}

Vehicular ad hoc networks are self-organizing networks which are developed from moving vehicles and they are differentiated by very high speed and narrow degrees of mobility patterns. The performance of vehicles in the network gets affected for various mobility models when applying to different environments. This part of the work discussed about various mobility patterns with respect to various road topologies. [2] [5] these papers proposed guideline for the generation of vehicular mobility models. It showed various approaches, for the vehicular mobility model development and their communication with network simulators for the peoples of different communities. Using these instructions to easily understand and objectively compare the different models, and eventually recognized the suitable one based on their requirements. [13] Classified the most pertinent vehicular propagation and channel models, with a particular focal point on the usability of the models for the evaluation of protocols and applications. [3] Discussed and analyzed about various mobility models for picky 
scenario using mobility simulators like MOVE (MObility model generator for VEhicular networks) and CityMob.[4]This author described about various mobility models like Highway, Manhattan and Freeway mobility models with respect to various routing protocols. Result analysis part demonstrated how performance of proposed scenario's routing protocol changes significantly, according to mobility models. Nabeel discussed about two different data sources and identify more realistic traffic flows along the California road way [1]. The authors use the PeMs database and associate the realistic data with the topology for the analysis purpose. Jin Woo Ro et al., [6] introduced new compositional carfollowing model which incorporated three distinct human factors based on hybrid input output automata (HIOA). Their model compared with various models IDM and FVDM and analyzed estimation error, reaction delay, and temporal anticipation. [7] yangliu et al., expressed cooperative adaptive cruise control and cooperative crash avoidance systems. This work addressed the problem through an overall objective function of CFG and velocity considering the safety hazard and the probability of cut-ins by other vehicles. [8] MofanZhou et al., proposed the new model for AVs (autonomous vehicles) and they analyze the performance with different AVs ratios. Upon analyzing, as the percentage of AVs increases, the RTT time gets reduced gradually.

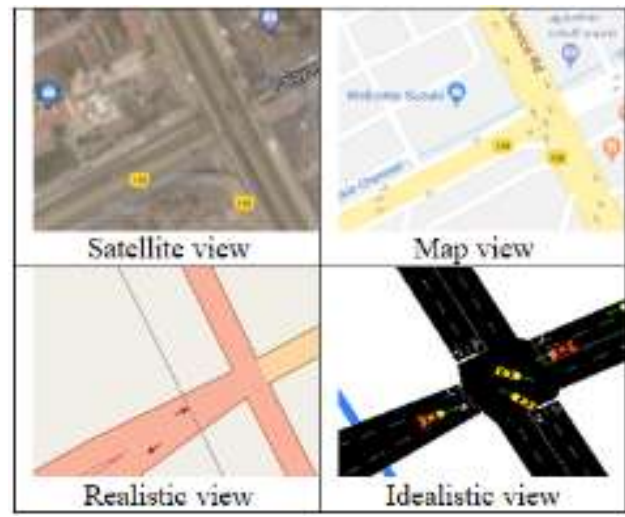

Fig. 1: Different Views of proposed topology

[9] It used different connectivity metrics such as lobby index, betweenness centrality and local clustering coefficient for analysis of the influence of mobility models based on the topological characteristics of a VANET. [10] [11] these papers proposed a solution for problem of lane changing using autonomous driving on highways using Scenario Model Predictive Control (SCMPC) mechanism and game-theoretical approach respectively using SUMO and NS2.

\section{Proposed work}

Vehicular Ad hoc Networks (VANETs) will be a sub division of implementing the pervasive environment. In order to achieve such big ad-hoc atmosphere through VANET, efficient mobility modelling for Vehicular Ad hoc Networks is highly demanding and firm task. It is difficult to meet out due to the distinctiveness of the network topology. The performance of mobility models depends on various factors such as speed, behaviour of drivers, road topology and obstacles. It is critical to ensure reliable data transmission in the presence of high speed vehicles. This scenario has motivated to design the new mobility model using real world topology collected from the various data sources. The proposed work consists of four phases that were explained in the following sections.

\subsection{Mobility Modelling}

The first phase of this work describes about the various mobility models in VANET and then compare the various data sources. In the third phase, proposed to design the suitable mobility models for the high speed multilane highway environment and then analysed its performance using suitable metrics.

\subsubsection{Krauss Model}

Krauss model is one of the car following model in which there will be a leader vehicle and the following vehicle. The following vehicle will maintain a certain distance to the leader vehicle and will also maintain a safe speed to adopt the behavior of the vehicle ahead. It considers that $\mathrm{u}$ is the maximum acceleration of vehicle $\left(\mathrm{m} / \mathrm{s}^{2}\right), \mathrm{v}$ is the maximum deceleration of the vehicles $\left(\mathrm{m} / \mathrm{s}^{2}\right), X_{\max }$ is the maximum speed of the vehicle $(\mathrm{m} / \mathrm{s}), \mathrm{k}$ is the length of vehicle $(\mathrm{m}), \alpha$ is the proficiency of the driver $(\alpha \in(0,1))$. The safe speed in the model is as shown below.

$\mathrm{X}_{\text {safe }}=\mathrm{X}_{\mathrm{t}}(\mathrm{t})+\frac{\mathrm{s}(\mathrm{t})-\mathrm{X}_{\mathrm{t}}(\mathrm{t}) * \tau}{\left(\mathrm{X}_{\mathrm{t}}+\mathrm{X}_{\mathrm{f}}\right) /(2 * \mathrm{v})+\tau}$

Where at time $t$ the leader vehicles speed is $x_{t}(t), x_{f}(t)$ is the following vehicle's speed at time $t, s(t)$ is the inter vehicle space of the following and the vehicle ahead. $\tau$ is the driver response time. The safe speed calculation does not consider the speed limit and the situation of acceleration in equation (1), so the correct speed must take the minimum value of three values.

$\mathbf{X}_{\mathrm{des}}=\min \left[\mathbf{X}_{\text {max }}, \mathbf{X}+\mathrm{u}^{*} \mathrm{t}_{\mathrm{k}}, \mathbf{X}_{\text {safe }}\right]$

It is considered that the driver do not have enough proficiency to adjust the vehicle to correct speed and also guarantees the vehicle speed while driving must be greater than zero. So the Krauss model formula is:

$$
\mathrm{x}(\mathrm{t})=\max \left[0, \mathrm{x}_{\mathrm{des}}-\alpha * \mathrm{u} * \operatorname{rand}(\mathrm{)}]\right.
$$

\subsubsection{Fluid Traffic Model}

The fluid traffic model shows the relation between the speed and the out flows of vehicles as the lambda shaped curve. It is based on the phenomena that, when the inflow rate and the vehicle density is increased the out flow rate grows linearly. The FTM shows the speed as steadily decreasing function of vehicular density and when the traffic rate touches the critical state, the speed will be at lower bound according to the equation (4)

$\mathrm{OS}=\max \left[\mathrm{S}_{\text {min }}, \mathrm{s}_{\max }\left(1-\frac{\mathrm{N}}{\mathrm{N}_{\mathrm{jam}}}\right)\right]$

Where OS is the output speed, $s_{\min }$ and $s_{\max }$ are the minimum and maximum speed, respectively, $\mathrm{N}_{\mathrm{jam}}$ is the vehicular density for which a traffic jam is detected, and $\mathrm{N}$ is the current vehicular density of the road the respective node is moving on. The current vehicle density is calculated by the formula $\mathrm{N}=\mathrm{n} / \mathrm{l}$, where $\mathrm{n}$ is the number of cars on the road and 1 is the length of the road segment itself. According to this model, cars traveling on crowded lane are forced to slow down, possibly to the minimum speed, if the vehicular density is found to be higher than or equal to the traffic jam density.

\subsubsection{Proposed Lane Changing Model}

The proposed lane changing model is to stimulate the multilane freeway traffic based on the microscopic traffic model. In this work, it uses the intelligent driver behavior as the underlying factor and guarantees the crash free driving. The lane changing behavior not only depends on the lane changing factors and the behavior of car, but also depends on the intellectual behavior of drivers. The lane change is performed, when it is favorable and 
safe. The parameters of the driver behavior are discussed as follows. The acceleration a of each vehicle $\beta$ is the continuous function of velocity $\mathrm{a}_{\beta}$, the net distance gap is $\mathrm{g}_{\beta}$ and the difference in velocity is $\Delta \mathrm{a}_{\beta}$ to the leading vehicle.

$\left.a \beta=v\left[1-\left(\frac{a \beta}{a_{0}}\right)^{4}-\left(\frac{g *\left(a_{\beta}, \Delta a \beta\right.}{g_{\beta}}\right)\right)^{2}\right]$

The position of the acceleration is defined in the following equation $\left.\dot{\mathrm{a}}_{\text {free }}(\mathrm{a})=\mathrm{a}\left[1-\left(\mathrm{a} / \mathrm{a}_{0}\right)^{4}\right]\right)$ on a free road and the braking conditions for deceleration is $\dot{\mathrm{a}}_{\text {int }}(\mathrm{g}, \mathrm{a}, \Delta \mathrm{a})=-\mathrm{a}\left(\mathrm{g}^{*} / \mathrm{g}\right)^{2}$, when the vehicle $\beta$ comes too close to vehicle ahead. The deceleration factor relies on the ratio of the minimum gap and the factual gap $\mathrm{g}_{\beta}$.

$$
\mathrm{g}^{*}(\mathrm{a}, \Delta \mathrm{a})=\mathrm{g}_{0}+\mathrm{aL}+\frac{\mathrm{a} \Delta \mathrm{a}}{2 \sqrt{\mathrm{xy}}}
$$

The minimum distance $\mathrm{g}_{0}$ in the dense traffic is for low velocities only. The stationary traffic is defined as the term aL, has the safe time gap L from the leading vehicle. The last term is active on non-stationary traffic and implements the intelligent driver behavior that includes the braking strategy, limits braking decelerations to the comfortable deceleration factor $y$.

The Krauss mobility model focuses mainly on the leader vehicle and their behaviors; they will maintain a safe distance between the vehicles. It shows low performance for the high speed and low density environment. Fluid traffic model will show the relation between the speed and the vehicles density on the single lane environment and while applying it to the multilane environment it lacks its performance rate. So the work proposes a new mobility model based on the intelligent driver behavior with lane changing for the proposed scenario.

\subsection{OSM Based Modelling}

Open Street Map is an open access database, which has all information regarding the location and attributes of every geographic feature. The proposed road topology is obtained using the OSM data and the generated files are feed as input to the mobility simulator as shown in Figure 2.The resultant trace file has been given as input to the network simulator for further analysis work in terms of mobility models.

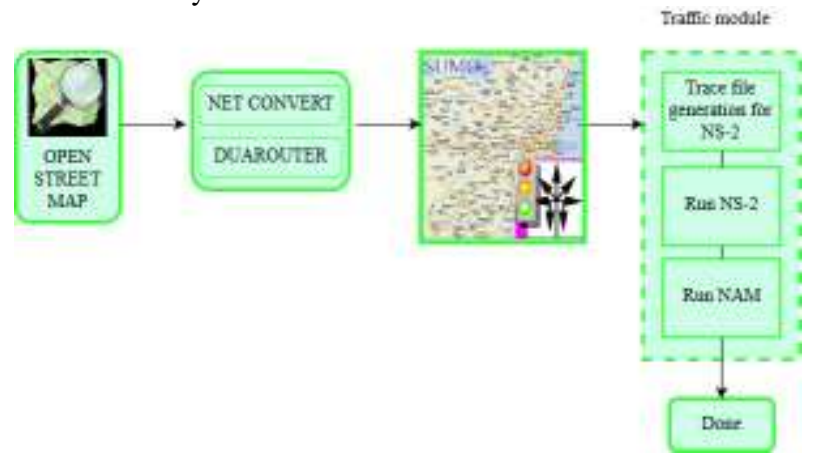

Fig. 2: Mobility modeling with OSM

\subsection{HTMS Based Modelling}

Highway traffic management system [15] uses the Automatic Traffic Counter and Classifier (ATCC) to collect the real time data from the highways in India. It detects, counts and classifies all the traffics passing. It also measures the speed of the vehicles, queues and classifies the vehicles. For this proposed work, the real time data of required road topology are collected from HTMS. Those collected data are feed as input to the mobility simulator to produce real time road topology as shown in Figure 3. The output of this phase is compared with the previous modeling phase and re- sult is analyzed using the network simulator in terms of various mobility models.

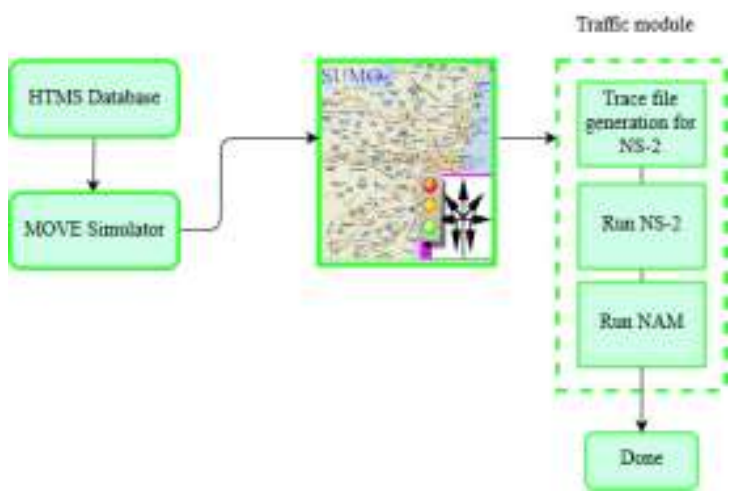

Fig. 3: Mobility modeling with HTMS

\subsection{Realistic Mobility Modelling}

From the above modeling phase, it obtains the OSM and the HTMS based data traces from the network simulator are used for further analysis. The analysis part takes place with the help of the different mobility models.

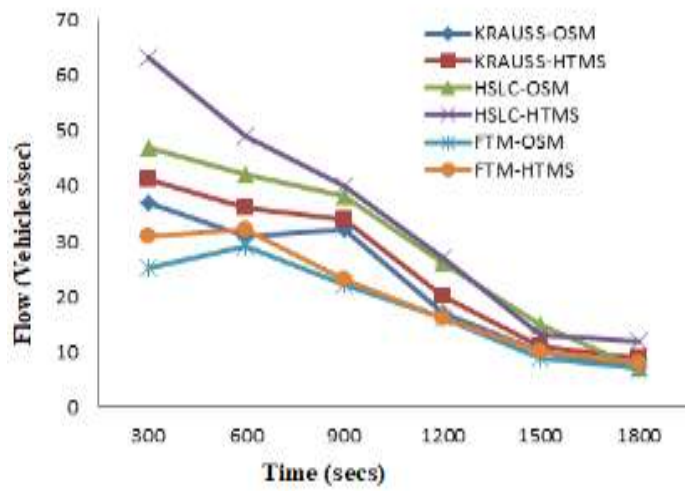

Fig.4: Time vs Flow

This work considers the proposed multilane highway as the road topology and the data's regarding the highway section are collected from the realistic data sources. While considering the flow and the average speed of the vehicles with respect to the time, Figure 4 $\& 5$ depicts that the mobility of the vehicles in the OSM data performs closely related to the HTMS data but HTMS data outperforms and provides the realistic performance to proposed road topology with respective to the various mobility models.

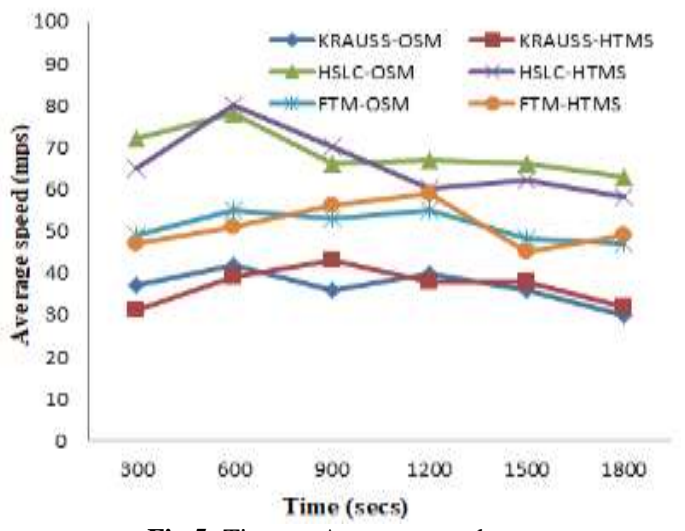

Fig.5: Time vs Average speed

Hence the HTMS data sources give the realistic data for high density and cross section environment and it has been used further for the future work of this paper. 


\section{Performance Analysis}

From the above analysis based on the realistic data sources, this proposed work uses multilane highway environment with the HTMS data as the input for simulating the commonly used mobility models and the proposed model. The simulation uses the AODV protocol for vehicle communication.

The PDR is defined as the ratio of number of received packets to the number of generated packets. For a large scale environment setup, this work determines the efficient mobility models by measuring PDR over number of vehicles. The simulation time is 1800s. The following graph shown in Figure 6 illustrates the proposed mobility model retained higher PDR of approximately $89 \%$ when number of vehicles ranges over 30 .

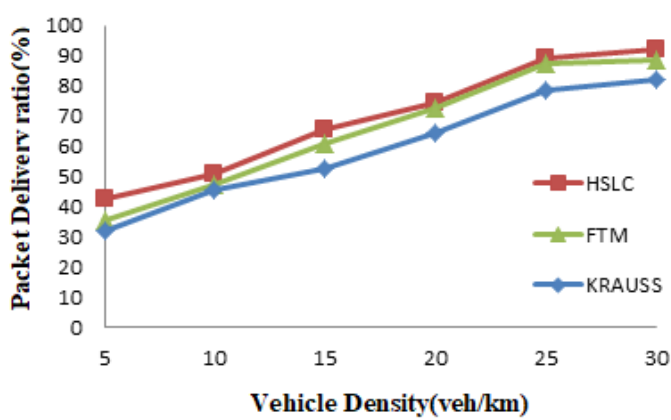

Fig. 6: The packet delivery ratio

The end to end delay is defined as the average time taken for a packet to traverse in the network. The delay is said as the total time taken by the packets to reach from source to destination and they are expressed in seconds. The following graph shown in Figure 7 illustrates the end to end delay of Krauss, fluid traffic and high speed lane changing mobility models. It is shown that the Krauss model and the proposed model experience the gradual decrease in end to end delay when the number of vehicles ranges from 5 to 10 .As the number of vehicles increases, the proposed model retains the lower delay of approximately $85 \%$ when compared with the other mobility models.

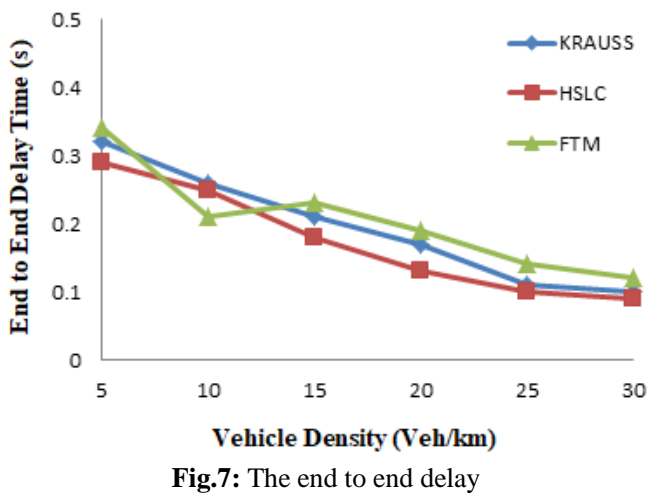

\section{Conclusion}

This work analyzed the proposed high speed-highway topology features based on the open street map and Highway Traffic Management system (HTMS) data sources. These data sources are given as input parameters to the traffic simulators and analyzed with respect to various mobility models. From this performance analysis, HTMS data source provided the realistic data. The performance of this proposed high speed lane changing model is compared with synthetic and behavioral models in terms of network management parameters. As the future work, it has planned to enhance the connectivity among the vehicles in the multilane highway environment.

\section{References}

[1] NabeelAkhtar Sinem Coleri Ergen and Oznur Ozkasap, "Vehicle Mobility and Communication Channel Models for Realistic and Efficient Highway VANET Simulation", IEEE Transactions on Vehicular Technology, Volume 64, no. 1, January 2015.

[2] Jerome Harrietal, "Mobility Models for Vehicular Ad Hoc Networks: A Survey and Taxonomy", IEEE communications surveys $\&$ tutorials, Volume 11, no. 4, fourth quarter 2009.

[3] BahidjaBoukenadil and Mohammed Feham, "Importance of realistic mobility models for VANET network simulation", International Journal of Computer Networks \& Communications (IJCNC) Volume 6, no.5, September 2014.

[4] B. Ramakrishnan, R. Bhagavath Nishanth, M. Milton Joe, R.S. Shaji "Comprehensive analysis of Highway, Manhattan and Freewaymobility models for vehicular ad hoc network", International Journal of Wireless and Mobile Computing Volume 9, Issue $1,2015$.

[5] Sarah Madi, Hend Al-Qamzi,“ A Survey on Realistic Mobility Models for Vehicular Ad Hoc Networks (VANETs)", IEEE international conference on networking, sensing and control (ICNSC), 2013.

[6] Jin Woo Ro, Partha S. Roop, Avinash Malik, Prakash Ranjitkar, "A Formal Approach for Modeling and Simulation of Human CarFollowing Behavior", IEEE Transactions on Intelligent Transportation Systems, Volume: PP, Issue: 99, October 2017.

[7] Yangliu Dou Daiheng Ni, Zhao Wang, Fengjun Yan, Jianqiang Wang, "Strategic car-following gap model considering the effect of cut-ins from adjacent lanes", IET Intelligent Transport Systems, Volume 10, Issue 10, December 2016.

[8] Mofan Zhou, Xiaobo Qu, Sheng Jin, "On the Impact of Cooperative Autonomous Vehicles in Improving Freeway Merging: A Modified Intelligent Driver Model-Based Approach", IEEE Transactions on Intelligent Transportation Systems, Volume 18 , Issue 6september2017.

[9] Senda Hammouda, Hajer Tounsi, Mounir Frikha, “ Towards a study of mobility models impact on VANET connectivity metrics", Third International Conference on The Network of the Future (NOF), Feb 2013.

[10] Glanluca Cesari, Georg Schildbach, Ashwin Carvalho, Francesco Borrelli, "Scenario Model Predictive Control for Lane Change Assistance and Autonomous Driving on Highways", IEEE Intelligent Transportation Systems Magazine, Volume: 9, Issue: 3, Fall 2017.

[11] Alireza Talebpour, Hani S. Mahmassani, Samer H. Hamdar,"Modeling Lane-Changing Behavior in a Connected Environment: A Game Theory Approach", Transportation Research Procedia, Volume 7, year 2015.

[12] Sourav Kumar Bhoi, Pabitra Mohan Khilar, "Vehicular communication: a survey", IET Journals and magazine, Volume 3, Issue: 3, jan 2014

[13] Wantanee Viriyasitavat, Mate Boban, Hsin-Mu Tsai, Athanasios Vasilakos, "Vehicular Communications: Survey and Challenges of Channel and Propagation Models", IEEE Vehicular Technology Magazine, Volume 10, Issue: 2May 2015

[14] Narendra Mohan Mittal, Savita Choudhary, "Comparative Study of Simulators for Vehicular Ad-hoc Networks (VANETs)" International Journal of Emerging Technology and Advanced Engineering, Volume 4, Issue 4, April 2014.

[15] Highway Traffic Management System (HTMS) http://www.metroinfrasys.com/ solution/highway-its/highwaytraffic-management-system/.

[16] Vaibhav Godbole, "Intelligent driver mobility model \& traffic pattern generation based optimization of reactive protocols for vehicular ad-hoc networks" International Journal of Information and Network Security (IJINS), Volume 2(3), pp. 207-214, Jun. 2013. 